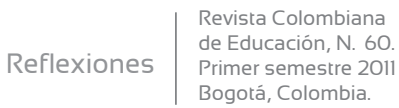

*

\section{Departamento de Comunicación Humana}

Facultad de Medicina

Universidad Nacional de Colombia

\title{
Aporte de la obra de Piaget a la comprensión de problemas educativos: su posible explicación del aprendizaje
}

\section{// A Contribution by Piaget's Work to Understanding Educational Issues A Probable Learning Explanation}

\author{
Nicolás Arias Velandia * \\ Rita Flórez Romero * *

\section{Resumen}

En este artículo se propone que la teoría de Piaget, como explicación del desarrollo cognitivo, sirve de base a una teoría del aprendizaje. Primero, se plantea que Piaget no se consideraba un investigador ni un teórico de la Educación, pero reconocía intereses comunes entre su proyecto intelectual y el de los investigadores en Educación. Luego, se plantea una posible explicación del aprendizaje de campos de conocimiento escolar usando planteamientos piagetianos. En tercer término se advierten las limitaciones de este proyecto, por medio de la diferencia entre el estudio básico del aprendizaje y el estudio del aprendizaje en el aula de clases. Al final se interpretan estos planteamientos a la luz de la relación entre "sujeto y objeto de conocimiento".

\section{Abstract}

This paper proposes Piaget's theory as an explanation of cognitive development as ground for a learning theory. Firstly, it suggests that Piaget did not consider himself as a researcher nor a theorist of education but instead, he had a common interest between his intellectual project and educational researchers'. Then, it proposes a possible explanation on learning of school knowledge fields using Piaget's approaches. Thirdly, restrictions of this project are evident by difference between the basic learning study and the classroom learning study. Finally these approaches are interpreted according to the relationship 'subject-object knowledge'.

\section{Palabras Clave}

Piaget, teorías del aprendizaje, aprendizaje escolar, sujeto y objeto de conocimiento.

\section{Keywords}

Piaget, learning theories, school learning, subject-object knowledge. 


\section{Introducción}

Al recorrer la obra de Piaget, Ilama la atención que algunos de sus comentaristas y críticos asumen dos posiciones extremas sobre su teoría en relación con la educación: la de aquellos que consideran que Piaget es un pedagogo o un autor en Educación, porque su obra trata del crecimiento de los mecanismos que nos permiten acceder al conocimiento (Aebli, 1958; Coll, 1987) y la de quienes conciben la obra de Piaget como una reivindicación del "conocimiento espontáneo" (cualquiera que sea su definición) que es determinado por el estadio del desarrollo en el cual el niño se encuentra (Elkind, 1976, 1981). Es también común que los defensores de esta última postura vean las convenciones del mundo adulto como "coacciones" que "limitan dicha espontaneidad" en el conocimiento del niño. Nos encontramos entonces ante una serie de autores que asumen un "Piaget pedagogo" y otros que asumen un "Piaget antieducativo", o, al menos, un "Piaget antiadultos". Por ese motivo es importante volver a preguntarnos qué pensaba Piaget acerca de diversos aspectos de la Educación y qué consecuencias tiene su teoría para pensar en asuntos de interés para la Educación.

Existen dos textos en los cuales este autor formula explícitamente una postura sobre asuntos educativos: A dónde va la educación (Piaget, 1975), texto realizado para la Unesco en 1972, por ser considerado una autoridad mundial en desarrollo de la inteligencia y el conocimiento. El otro es el primer capítulo de Problemas de psicología genética (Piaget, 1976), en el cual se postulan implicaciones en la consideración de factores endógenos y exógenos en el desarrollo humano. Este último parece escrito en respuesta a dudas y críticas a sus planteamientos sobre la relación entre la interacción social y la formación del conocimiento.

Al realizar esta exploración, se constata que lo planteado explícitamente por Piaget sobre asuntos educativos tiene matices de prudencia, en los cuales resalta que él realiza investigación psicológica y que no es un pedagogo (Piaget, 1976). Exhorta al lector a tomar su punto de vista solamente como el de una "opinión informada" sobre el desarrollo infantil y del conocimiento. También, evita lanzar directrices definitivas. Apenas sugiere algunos temas que considera de interés para su teoría y para la investigación educativa (Piaget, 1976; Piaget y García, 1983). 
Sin embargo, el conjunto de la obra de Piaget también contiene sugerencias sobre cómo se desarrolla el conocimiento de los niños en diferentes áreas. De alguna manera, el interés último de algunos objetos de estudio en la investigación educativa es el mismo que en Piaget: saber cómo crece el conocimiento de las personas en diferentes áreas para poder mejorar la educación de niños y jóvenes especialmente (Gardner, 2000; Hederich, 2007; Bruer, 1997). En consecuencia, un aporte crucial de la teoría de Piaget a la investigación educativa es la idea del conocimiento como un proceso de reconstrucción, y que dicha idea aporta a la comprensión del aprendizaje así como ha sido útil para comprender el desarrollo cognitivo.

En primer lugar, en este texto se exponen las ideas de Piaget que sirven para pensar una teoría del aprendizaje, con apoyo adicional de planteamientos de investigadores contemporáneos en educación. Luego, se exponen algunas limitaciones de estos planteamientos. Finalmente, se presentan algunas conclusiones.

\section{El aporte de Jean Piaget: ideas para una teoría del aprendizaje}

Piaget no planteó que el objetivo de su trabajo teórico o investigativo fuese la Educación o la Pedagogía; sin embargo, se reconoce en este autor un gran aporte a la manera de entender la instrucción y el conocimiento, y a la concepción del educando como una persona con algún tipo de conocimiento, incluso desde antes de haber recibido enseñanza escolar en algún campo (Puche, 2001; Flórez y Arias, 2007).

De otra parte, Piaget no formuló explícitamente una teoría del aprendizaje y solamente se le reconoce como un autor central en temas de desarrollo cognitivo (Hederich, 2007; Torrado, 1997). No obstante, se han desarrollado programas investigativos a partir de la aproximación piagetiana (Ferreiro y Teberosky, 1979, 1999; Karmiloff Smith, 1994; Bates, 1979) que han formulado hipótesis sugerentes acerca de cómo aprenden los niños y los jóvenes en algunos campos (Ferreiro, 2007a, 2007b). Por lo tanto, es legítimo pensar que su teoría aporta a la comprensión de problemas de interés para el aprendizaje en la Educación.

Piaget (1976) concibió el conocimiento como un proceso de consolidación y elaboración de la acción del sujeto sobre el mundo y no solamente como un acervo, y como un proceso que se inscribe en el tiempo. El conocimiento entonces tiene un tiempo, entendido en dos sentidos: como duración, tiempo cronológico que toma el desarrollo de alguna capacidad o momento de aparición de la misma en el crecimiento del niño, y como sucesión, tiempo en el cual se marca una secuencia en la cual ciertos logros del conocimiento 
aparecen luego de algunos y antes que otros, porque siguen una secuencia lógica de consolidación (Piaget, 1976).

Esta distinción sirve para aclarar por qué muchos cambios en el desarrollo no son inmediatos ni de aparición súbita. La duración como la sucesión en estadios, etapas y fases de desarrollo no siempre siguen un patrón uniforme de crecimiento, ya que dependen de la maduración orgánica, de la experiencia de la persona con objetos del mundo físico, de la experiencia de la persona con el mundo social y de la equilibración (Piaget, 1976). El sujeto articula los logros previos y los logros posteriores en su conocimiento, construido a partir de la búsqueda de explicaciones cada vez más abarcadoras y consistentes sobre el mundo.

Se suele ubicar a Piaget como un teórico individualista del desarrollo y a Vygostki como un teórico más "social" de este fenómeno o que "reconoce más el papel de la interacción social" (Cf., Rodríguez y Moro, 1998). Sin embargo, Piaget (1975, 1976) también resalta la importancia de la experiencia social y el papel de los entornos adultos ricos en la consolidación del desarrollo de los niños. En este sentido, el conocimiento para Piaget tiene como fuente el modelo cultural del mundo adulto (Piaget y García, 1982); por lo tanto el conocimiento cambia, se desarrolla y no tiene como única característica organizarse en estadios (Cf., Piaget, 1976). Con sus mismas palabras, este autor afirma:

Bien pronto, en la experiencia del niño, las situaciones con las cuales se enfrenta son generadas por su entorno social, y las cosas aparecen en contextos que les otorgan significaciones especiales. No se asimilan objetos "puros". Se asimilan situaciones en las cuales los objetos desempeñan ciertos papeles y no otros. Cuando el sistema de comunicación del niño con su entorno social se hace más complejo y más rico, y particularmente cuando el lenguaje se convierte en medio dominante, lo que podríamos llamar la experiencia directa de los objetos comienza a quedar subordinada, en ciertas situaciones, al sistema de significaciones que le otorga el medio social. El problema que aquí surge para la epistemología genética es explicar cómo queda la asimilación, en dichos casos, condicionada por tal sistema social de significaciones y en qué medida la interpretación de cada experiencia particular depende de ellas (Piaget y García, 1982, p. 228). 
En consecuencia con lo anterior, Piaget (1976) plantea que el desarrollo de algunos niños se retrasa por cierta pasividad del mundo adulto alrededor que impide que el niño acceda a todas las herramientas de su conocimiento. Dado que el planteamiento piagetiano admite este importante papel del entorno social en la promoción del desarrollo y de los cambios en el conocimiento como aspectos característicos del desarrollo, entonces se puede pensar desde allí una posible teoría del aprendizaje.

Otros autores contemporáneos observan que algunas capacidades se desarrollan con un patrón similar en la mayoría de niños durante los primeros tres años de vida, como el caso de: lenguaje, percepción, organización y clasificación de eventos del mundo e interacción con otras personas, tal vez con la excepción de los niños sometidos a condiciones extremas de deprivación, y que otras dependen más del privilegio que se da a ciertos conocimientos en cada entorno particular ${ }^{1}$, por ejemplo: reflexiones sobre el lenguaje, control y monitoreo de la acción, conocimientos sobre notaciones gráficas, taxonomización y matematización de fenómenos, y aspectos pragmáticos de la interacción comunicativa (Gardner, 2000;

\footnotetext{
El primer conjunto de estas capacidades suele ser estudiado como fenómenos del desarrollo temprano y el segundo conjunto se estudia habitualmente como fenómenos de aprendizaje. El tránsito de las primeras a las segundas suele ubicarse cerca del tercer año de edad del niño.
}

Catts y Kamhi, 2005; Karmiloff Smith, 1994; Karmiloff y Karmiloff Smith, 2005; Keil, 1979; Gelman y Gallistel, 1986; Flórez, Restrepo y Schwanenflugel, 2007).

De acuerdo con esta distinción, la obra de Piaget ha servido a autores posteriores para conceptualizar procesos de aprendizaje (Ferreiro, 2007b). Se han planteado extensiones de esta teoría al trabajo con objetos culturales, es decir, con actividades que solamente se realizan y se privilegian en algunos entornos sociales. Se puede citar el trabajo de E. Ferreiro en la comprensión del aprendizaje de la lengua escrita, sobre el cual plantea lo siguiente:

La indagación psicológica cuidadosa nos lleva a concluir que los niños comienzan desde mucho antes de lo que la escuela imagina a ocuparse de la escritura; que el trabajo cognitivo de la escritura también contribuye al desarrollo operatorio; que además de los problemas lógicos de carácter general que hemos mencionado, hay problemas específicos que este objeto particular plantea, particularmente en su relación con el lenguaje, que precisamente por tratarse de un objeto simbólico, la interacción con los intérpretes no es un mero agregado, sino que es constitutiva de la posibilidad de comprensión del objeto.

(...) La demostración de que la escritura se constituye en objeto de conocimiento en el curso de 
desarrollo, y cambia probablemente la relación del sujeto con su lengua, es contraria a la idea comúnmente admitida según la cual la escritura es una simple técnica de transcripción. Esta nueva concepción está cargada de consecuencias pedagógicas (...). La comprensión del sistema de escritura como tal, es ya un gran logro. Pero eso solo no basta. La escuela debe permitir un acceso inteligente a las condiciones de enunciación propias de la escritura: hay que aprender a solicitar, argumentar, contar, dar instrucciones, preguntar, responder, informar, comentar y dialogar por escrito (Ferreiro, 2007a, p. 407).

Sobre el conocimiento de objetos culturales se pueden plantear secuencias de logro similares a las planteadas por Piaget (1976) en un sentido particular: los logros previos son soporte y condición necesaria para pasar a los logros siguientes (Piaget, 1976; Cf., Koffka, 1973).

El conocimiento de objetos culturales se entiende entonces como un proceso de aprendizaje ${ }^{2}$, se refiere al conocimiento relacionado con objetos, eventos y actividades propias de un grupo cultural o de un contexto específico y que no se da necesariamente en otros contextos (Ferreiro, 2007a). El niño o el aprendiz tienen desde el principio ideas y conceptos, que son también parte de su conocimiento, y hacen parte de un marco orientador de las acciones. Dicho marco cambia en cada momento de comprensión. De acuerdo con ideas inspiradas en Piaget (1976), se trataría de conocimientos que presentan grandes variaciones en las edades de aparición en los niños, tienen momentos de continuidad y de saltos súbitos, y pueden variar en función del privilegio diferencial de ciertos conocimientos en el entorno cultural de la persona. A pesar de esto, también mantendrían una secuencia general invariante de logros en el aprendiz (Cf., Piaget, 1975; Ferreiro, 2007a).

Como muestra de esta idea, se presentan los trabajos de Emilia Ferreiro, sus colegas y colaboradores en torno a los sistemas de escritura (Ferreiro, 1999) y el enfoque de trayectorias de aprendizaje de Clements y Sarama (2009) en el aprendizaje inicial de las matemáticas. El primero plantea una secuencia específica en la comprensión de la función de la escritura y del lenguaje escrito, y el segundo plantea una secuencia que sigue el apren-

2 El aprendizaje se define acá como el proceso de adquisición de conocimientos o habilidades y su consolidación en la memoria, cuando dichos conocimientos no pertenecen a un logro universal esperado en todas las personas. 
dizaje inicial de las Matemáticas en niños preescolares. Ambos plantean que esta secuencia solo se despliega óptimamente ante actividades con sentido propuestas por el adulto al niño en la interacción social planeada. Así, según Emilia Ferreiro:

Para lograr esa comprensión analítica entre las marcas escritas y la lengua oral, hace falta esa actividad estructurante del sujeto que Piaget describió en otros dominios del conocimiento. Es esencial también recibir información específica, solo que esa información será asimilada por el sujeto en desarrollo a sus esquemas conceptuales. $\mathrm{Y}$ hace falta una prolongada interacción con el objeto a ser conocido, un objeto que se presenta a través de muchos otros objetos. La escritura no existe en el vacío. Las superficies que la cultura ha construido para ser portadoras de marcas escritas tienen nombre y función: se llaman periódicos, libros, calendarios, documentos de identidad, diccionarios, anuncios, envases de alimentos o medicinas, carteles urbanos con nombres de calles, indicaciones para vehículos y peatones, propaganda comercial... La lista es muy larga de establecer.

La escritura existe en contexto. El pizarrón escolar es apenas uno de los contextos posibles: el más analítico pero no el más funcio- nal. Los niños urbanos, rodeados de escritura desde que nacen, no siempre están rodeados de informantes e intérpretes. Todos ellos, incluso los hijos de analfabetos, logran aprender bastante sobre esas marcas, gracias a sus esfuerzos activos por compararlas, ordenarlas y reproducirlas. Pero son muchos también los que llegan a la escuela sin haber sido destinatarios de un acto de lectura en voz alta, sin haber enfrentado el desafío del misterio, sin haber traspasado el umbral de la opacidad de las marcas. (...) (Ferreiro, 2007a, p. 401).

Esta cita extensa de Ferreiro pone de manifiesto un elemento que es distintivo de los actos educativos: la presencia del adulto que guía la interpretación de los niños, o del enseñante que guía la interpretación de los aprendices, en actos que tiene sentido dentro de un medio cultural particular (Puche - Navarro, 2001). Con frecuencia, estos actos logran un efecto en el aprendizaje cuando se constituyen como una especie de interfase o espacio transicional que sirve al niño para pasar de lo conocido a lo desconocido, mediante el desarrollo de actividades que son planeadas por quien enseña pero donde el aprendiz tiene un espacio para desplegar su propias posibilidades de acción, de razonamiento y de conocimiento previamente consolidado (Puche - Navarro, 2001; Ferreiro, 2007a; Flórez, Arias y Castro, 2010). De allí que, todos 
los niños elaboren conocimientos sobre la lengua escrita, pero solo algunos tengan la oportunidad de verla como un objeto de conocimiento en su vida por las oportunidades que reciben del contexto de los adultos.

Las interfases generan un contexto en el cual los niños y los aprendices pueden desplegar su razonamiento, porque el curso de sus mismas acciones se lo exige. Algunas interfases o espacios transicionales encontrados por nuestro propio equipo de investigación son: el uso de operaciones metalingüísticas y metacognitivas (Flórez, Torrado y Mesa, 2006; Flórez y cols., 2005), actividades de lectura conjunta (Flórez, Torrado y Arias, 2006) y actividades que implican el razonamiento sobre acontecimientos e intenciones de los agentes que participan en ellas (Symons y cols., 2005).

Con estas ideas, es posible concretar la opinión de Piaget (1975) de que la educación logra su cometido cuando en los individuos hay "adquisición de herramientas y formas de razonamiento que permitan dar forma al conocimiento específico en sus diferentes fases y de manera sostenida en el tiempo" (p. 15).

\section{Limitaciones y "usos buenos y malos" de una teoría del aprendizaje basada en Piaget}

Con esta propuesta deben advertirse limitaciones. No se está planteando una transferencia directa de los hallazgos de laboratorio o de investigaciones básicas a las aulas de clase, o a espacios similares de enseñanza y aprendizaje. Lo que se propone en este texto es un marco para desarrollar investigaciones en dos campos diferentes pero relacionados: el estudio del proceso de aprendizaje en distintos campos de conocimiento, y el estudio del desarrollo de conocimientos en un aula o contexto particular de enseñanza y aprendizaje (cf., Larreamendy-Jöerns, 2002).

En este punto adherimos a la postura de la psicología cognitiva norteamericana interesada en procesos de aprendizaje en educación (Larreamendy-Jöerns, 2002). Así, tendríamos un campo de trabajo que se ocupa del estudio básico del aprendizaje en diferentes ámbitos de conocimiento, que trabaja en las características y capacidades de los aprendices, una muestra de ello es capacidad de esquematización en expertos en física y economía, modelos explicativos de principiantes en biología sobre la teoría de la evolución, en el conocimiento desarrollado por expertos en un área o metas de aprendizaje, por ejemplo, esquema de ope- 
ración de físicos expertos al abordar problemas de electromagnetismo y en el proceso de aprendizaje o de paso de conocimiento ingenuo a uno más próximo al del experto en un área, como evolución de las explicaciones de acontecimientos particulares en estudiantes de historia y en estudiantes de secundaria. Otro campo de trabajo planteado es el aprendizaje de campos de conocimiento en el aula, que se refiere a diseño de situaciones para promover el aprendizaje en un área particular, que implica el trabajo de personas que diseñan tareas o secuencias de las mismas y condiciones del contexto particular de los educadores, de los aprendices, de su entorno y del campo disciplinar en el cual se desarrollará el aprendizaje.

Esta aclaración es pertinente porque, como expone Larreamendy-Jöerns (2002) en el pasado era común transferir hallazgos de investigaciones psicológicas de contextos como el laboratorio o la investigación básica, al contexto del aula de clase, tanto en interpretaciones de la teoría de Piaget (Cf., Aebli, 1958) como en otros planteamientos psicológicos, para aplicarlos en la enseñanza. Como consecuencia de esto, hubo una tendencia muy fuerte a realizar transferencias directas de hallazgos psicológicos a las aulas de clase, que con frecuencia ignoraron la especificidad del conocimiento disciplinar y los contextos educativos particulares (Colomina, Onrubia y Rochera, 2001).
De otra parte, si se deriva una teoría del aprendizaje desde Piaget, esta no puede reducir a priori la posible competencia del niño a una clasificación de su logro en los estadios o fases del desarrollo que planteó este autor. Por el contrario, nuestro punto de vista exige que podamos explicar hallazgos en diversos campos de conocimiento que el niño y el adulto enfrentan desde una visión piagetiana, para lo cual la investigación debe observar tanto las actividades, prácticas y experiencias del niño, o del aprendiz, como las restricciones que el objeto a conocer impone a la actividad (Ferreiro, 2007b). En el mismo sentido, reconocer también la influencia de formas de procesamiento de información más automáticas, a pesar de que el proceso de construcción de conocimiento del sujeto siga reconociéndose como rector de la evolución en el aprendizaje (Karmiloff - Smith, 1994).

\section{Conclusiones}

Un gran aporte de la obra de Piaget es el rescate de la epistemología natural, al suponer que detrás de toda operación hay conocimiento, así el conocimiento no esté ajustado a los cánones convencionales de conocimientos disciplinares. El punto que rescata Piaget en su obra es que el aprendiz se hace preguntas sobre su objeto de conocimiento y es además una persona cuyo razonamiento forma parte de la misma acción que realiza (Ferreiro, 2007b). Para Piaget el conocimiento es acción en proceso. 
Es posible entonces derivar de los planteamientos de Piaget ideas para formular una teoría del aprendizaje, que no existe en su obra de manera explícita, porque en el planteamiento piagetiano se enuncia una relación dialéctica entre sujeto y objeto en el conocimiento, en la cual hay aporte de la actividad del sujeto y también de características del objeto. En los conocimientos propios de contextos específicos, objetos culturales, las características de dicho objeto incluyen formas de uso convencional en una comunidad específica, que han de ser mostradas por el adulto o por la persona más experta. Una teoría del aprendizaje derivada de, o una extensión teórica de los planteamiento de Piaget, tiene algunas ventajas porque permite:

1.Entender el punto de vista del sujeto sobre el objeto de conocimiento, especialmente en aspectos en los cuales puede ser muy diferente del punto de vista convencional del conocimiento o de la disciplina que se aprende (Piaget, 1976).

2.Interpretar razonamientos de los aprendices y hallazgos sobre su desempeño a la luz de la naturaleza y del cambio histórico del objeto cultural en cuestión (Ferreiro, 2007a).

3.Abordar conceptual y empíricamente la manera en la cual la persona desarrolla su acción en el mismo momento de su ejecución y sus razonamientos subyacentes (cf., Puche, 2001).

\section{Referencias}

Aebli, H. (1958). Una didáctica fundada en la psicología de Jean Piaget. Buenos Aires: Kapelusz

Bates, E., Benigni, L., Bretherton, I., y Camaioni, A. (1979). The emergence of symbols: cognition and communication in infancy. New York: Academic Press.

Bruer, J. (1997). Escuelas para pensar. La nueva ciencia del aprendizaje en el aula. Madrid: Paidós Ibérica - Ministerio de Educación y Ciencia de España.

Catts, H. y Kamhi, A. (2005). The Connections between Language and reading disabilities. Mahwah, NJ: Lawrence Erlbaum. 
Clements, D. y Sarama, J. (2009). Learning and teaching early math. The learning trajectories approach. New York: Routledge.

Coll, C. (1987). Psicología y currículum. Barcelona: Laia.

Colomina, R., Onrubia, J. y Rochera, M. J. (2001). Interactividad, mecanismos de influencia educativa y construcción de conocimiento en el aula. En C. Coll, J. Palacios y A. Marchesi (comps.), Desarrollo Psicológico y Educación, Vol. 2: Psicología de la Educación Escolar, pp. 437-458. Madrid: Alianza.

Elkind, D. (1976). Cognitive development and reading. En $\mathrm{H}$. Singer y R. Rudell (eds.), Theoretical models and processes of reading. Newark, NJ: International Reading Association.

Elkind, D. (1981). Stages in development of reading. En I. Siegel, D. Brodzinsky y E. Golinkoff (eds), New directions in Piagetian theory and practice. Hillsdale, NJ: Lawrence Erlbaum.

Ferreiro, E. (1999). Cultura escrita y educación. México: Fondo de Cultura Económica. Entrevistas compiladas por Graciela Quinteros.

Ferreiro, E. (2007a). La adquisición de los objetos culturales: el caso particular de la lengua escrita. En E. Ferreiro (con el cuidado y apoyo de A. Soto), Alfabetización de niños y adultos. Textos escogidos, pp. 399 - 409. Pátz- cuaro (Edo. Michoacán), México: Centro de Cooperación Regional para la Educación de Adultos en América Latina y el Caribe CREFAL.

Ferreiro, E. (2007b). Aplicar, replicar, recrear. Acerca de las dificultades inherentes a la incorporación de nuevos objetos al cuerpo teórico de la teoría de Piaget. En E. Ferreiro (con el cuidado y apoyo de A. Soto), Alfabetización de niños y adultos. Textos escogidos, pp. 411 - 420. Pátzcuaro (Edo. Michoacán), México: Centro de Cooperación Regional para la Educación de Adultos en América Latina y el Caribe CREFAL.

Ferreiro, E. y Teberosky, A. (1979). Los sistemas de escritura en el niño. México: Siglo XXI.

Flórez, R. y Arias, N. (2007). Las representaciones mentales de los niños y las niñas y el aprendizaje inicial de la lectura. En F. A. Santamaría y M. H. Barreto (comps.), Lenguaje y Saberes Infantiles, pp. 159 - 165. Bogotá: Cátedra UNESCO en Desarrollo del Niño / Universidad Distrital Francisco José de Caldas.

Flórez, R., Arias, N. y Castro, J. (2010). Construyendo puentes: lectura y escritura en la educación inicial. En Fundalectura (ed.), ¡Los niños son un cuento! Lectura en la Primera Infancia. Memorias / $9^{\circ}$ Congreso Nacional de Lectura, 67 - 87. Bogotá: Fundalectura. 
Flórez, R., Restrepo, M. A. y Schwanenflugel, P. (2007). Alfabetismo emergente: investigación, teoría y práctica. El caso de la lectura. Bogotá: Departamento de Comunicación Humana, Universidad Nacional de Colombia - Instituto para la Investigación Educativa y el Desarrollo Pedagógico IDEP, Alcaldía Mayor de Bogotá, D. C.

Flórez, R., Torrado, M. C., Arévalo, I., Mesa, C., Mondragón, S. y Pérez, C. (2005). Habilidades metalingüísticas, operaciones metacognitivas y su relación con los niveles de competencia en lectura y escritura: un estudio exploratorio. Forma y Función, 18: 15 - 44.

Flórez, R., Torrado, M. C. y Mesa, C. (2006). Emergencia de las capacidades metalingüísticas. Revista Latinoamericana de Psicología. 38 (3): 457 - 475.

Gardner, H. (2000). La educación de la mente y el conocimiento de las disciplinas: lo que todos los estudiantes deberían comprender. Barcelona: Paidós.

Gelman, R., y Gallistel, C. R. (1986). The child's understanding of number. Cambridge, Mass: Harvard University Press.

Hederich, C. (2007). Estilo cognitivo en la dimensión independencia - dependencia de campo: influencias culturales e implicaciones para la educación. Bogotá: Universidad Pedagógica Nacional.

Karmiloff, K. y Karmiloff - Smith, A. (2005). Hacia el lenguaje: del feto al adolescente. Madrid: Morata.

Karmiloff - Smith, A. (1994). Más allá de la modularidad. La ciencia cognitiva en la perspectiva de desarrollo. Madrid: Alianza.

Keil, F. (1979). Semantic and conceptual development: an ontological perspective. Cambridge, Mass: Harvard University Press.

Koffka, K. (1973). Principios de Psicología de la forma. Buenos Aires: Paidós.

Larreamendy, J. F. (2002). Ciencia cognitiva y Educación. Más allá de la falacia de la aplicación. Diálogos, Discusiones en la Psicología contemporánea. 2: 139 - 151.

Piaget, J. (1975). A dónde va la educación. Barcelona: Teide.

Piaget, J. (1975). Problemas de psicología genética. Barcelona: Ariel.

Piaget, J., y García, R. (1983). Psicogénesis e historia de la ciencia. México: Siglo XXI. 
Puche - Navarro, R. (2001). De la metáfora del niño como científico a la racionalidad mejorante. En R. Puche - Navarro, D. Colinvaux y C. Dibar - Ure (comps). El niño que piensa. Un modelo de formación de maestros. pp. 23 - 55. Cali: Artes Gráficas del Valle (Volumen conjunto apoyado por Universidad del Valle, Universidad Federal Fluminense, Universidad de Buenos Aires, Ministerio de Educación Nacional de la República de Colombia y Organización de Estados Americanos).
Rodríguez, C. y Moro, C. (1998). El mágico número tres: cuando los niños aún no hablan. Barcelona: Paidós.

Symons, D. K., Peterson, C. C., Slaughter, V., Roche, J. y Doyle, E. (2005). Theory of mind and mental state discourse during book reading and storytelling tasks. British Journal of Developmental Psychology. 23, 81 - 102. 